\title{
Modulation of Glucose Regulation and Insulin Secretion by Circadian Rhythmicity and Sleep
}

Eve Van Cauter, ${ }^{\star \ddagger}$ John D. Blackman, ${ }^{*}$ Dianne Roland, ${ }^{*}$ Jean-Paul Spire, Samuel Refetoff, ${ }^{\star \prime}$ and Kenneth S. Polonsky* Departments of ${ }^{*}$ Medicine, ${ }^{8}$ Neurology, and $"$ Pediatrics, University of Chicago, Chicago, Illinois 60637; and

${ }^{\ddagger}$ Institute of Interdisciplinary Research, Université Libre de Bruxelles, Brussels, B-1070 Belgium

\begin{abstract}
To define the roles of circadian rhythmicity (intrinsic effects of time of day independent of the sleep or wake condition) and sleep (intrinsic effects of the sleep condition, irrespective of the time of day) on the 24-h variation in glucose tolerance, eight normal men were studied during constant glucose infusion for a total of $53 \mathrm{~h}$. The period of study included $8 \mathrm{~h}$ of nocturnal sleep, $28 \mathrm{~h}$ of continuous wakefulness, and $8 \mathrm{~h}$ of daytime sleep. Blood samples for the measurement of glucose, insulin, C-peptide, cortisol, and growth hormone were collected at 20-min intervals throughout the entire study. Insulin secretion rates were derived from $\mathrm{C}$-peptide levels by deconvolution. Sleep was polygraphically monitored.

During nocturnal sleep, levels of glucose and insulin secretion increased by $31 \pm 5 \%$ and $60 \pm 11 \%$, respectively, and returned to baseline in the morning. During sleep deprivation, glucose levels and insulin secretion rose again to reach a maximum at a time corresponding to the beginning of the habitual sleep period. The magnitude of the rise above morning levels averaged $17 \pm 5 \%$ for glucose and $49 \pm 8 \%$ for calculated insulin secretion. Serum insulin levels did not parallel the circadian variation in insulin secretion, indicating the existence of an approximate $40 \%$ increase in insulin clearance during the night. Daytime sleep was associated with a $16 \pm 3 \%$ rise in glucose levels, a $55 \pm 7 \%$ rise in insulin secretion, and a $39 \pm 5 \%$ rise in serum insulin.

The diurnal variation in insulin secretion was inversely related to the cortisol rhythm, with a significant correlation of the magnitudes of their morning to evening excursions. Sleep-associated rises in glucose correlated with the amount of concomitant growth hormone secreted. These studies demonstrate previously underappreciated effects of circadian rhythmicity and sleep on glucose levels, insulin secretion, and insulin clearance, and suggest that these effects could be partially mediated by cortisol and growth hormone. (J. Clin. Invest. 1991. 88:934942.) Key words: cortisol $\bullet$ growth hormone $\bullet$ counterregulatory mechanisms • insulin clearance • glucose tolerance
\end{abstract}

\section{Introduction}

In normal man, glucose tolerance varies with the time of day. Plasma glucose responses to oral glucose, intravenous glucose,

Address correspondence to Eve Van Cauter, Ph.D., Department of Medicine, Box 138, University of Chicago, 5841 South Maryland Avenue, Chicago, IL 60637.

Received for publication 6 February 1991 and in revised form 20 May 1991

J. Clin. Invest.

(c) The American Society for Clinical Investigation, Inc. 0021-9738/91/09/0934/09 \$2.00

Volume 88, September 1991, 934-942 or meals are markedly higher in the evening than in the morning (1-3). Diminished insulin sensitivity and decreased insulin secretion are both involved in causing reduced glucose tolerance later in the day $(2,4,5)$. Using experimental protocols involving intravenous glucose infusion at a constant rate for 24-30 hours, we have recently shown that glucose tolerance deteriorates as the evening progresses, reaching a minimum around the middle of the night $(6,7)$. This diurnal variation was not caused by changes in activity level since it persisted during continuous bed rest (7). Fluctuations in circulating levels of insulin or in insulin secretion rates did not consistently parallel the pattern of glucose changes. Since glucose concentrations began to increase in the late afternoon or early evening, well before bedtime, and continued to rise until approximately the middle of the night, both sleep-independent effects, reflecting circadian rhythmicity, and sleep-dependent effects could be involved in producing the overall 24 -h pattern.

The purpose of this study was to define the respective roles of circadian rhythmicity (intrinsic effects of time of day independent of the sleep or wake condition) and sleep (intrinsic effects of sleep per se irrespective of the time of day) in the 24-h variation of glucose tolerance. To differentiate between the effects of circadian rhythmicity and those of sleep, eight normal men were subjected to a 12-h delay of their sleep period. The rationale behind this experimental design was to allow the effects of time of day to be observed in the absence of sleep and the effects of sleep to be observed at an abnormal circadian time. Glucose was infused at a constant rate for $57 \mathrm{~h}$ to avoid confounding effects of prolonged fasting or meal ingestion. Blood samples were collected at 20-min intervals for the last 53 $h$ of the infusion. Sleep was polygraphically monitored. In addition to measurements of glucose, insulin, and C-peptide, the levels of cortisol, a counterregulatory hormone which is a robust marker of circadian rhythmicity, and those of growth hormone $(\mathrm{GH})^{1}$, a counterregulatory hormone profoundly modulated by sleep, were determined on each blood sample. Insulin secretion rates (ISR) were derived from plasma C-peptide levels by deconvolution.

\section{Methods}

Subjects. Studies were performed in eight healthy men, aged 22-27 yr. Mean \pm SEM body weight and body mass index were respectively $72 \pm 2$ $\mathrm{kg}$ and $22.7 \pm 0.7 \mathrm{~kg} / \mathrm{m}^{2}$. None of the subjects had a history of endocrine or psychiatric disorder. They were nonsmokers who were not taking any drugs. Shift workers or subjects who had travelled across time zones within $60 \mathrm{~d}$ before the study were excluded. All potential volunteers answered a detailed questionnaire concerning their daily routines,

1. Abbreviations used in this paper: DS, daytime sleep; GH, growth hormone; ISR, insulin secretion rates; NS, nocturnal sleep; NW, nocturnal wakefulness; REM, rapid eye movement; SP, sleep period; SW, slow wave. 
their sleep-wake cycle, and meal schedule. Those with irregular life habits or with sleep complaints were excluded.

Experimental protocol. The studies were carried out in the Clinical Research Center of the University of Chicago after written informed consent had been obtained. The experimental protocol was approved by the Institutional Review Board.

For one week before the study, the subjects complied with a standardized sleep schedule (2300-0700). To become habituated to the study conditions, the subjects slept in the sleep laboratory from 2300 0700 on the two days before the start of the study. On the first night, the electrodes for polygraphic sleep recording were attached but there was no actual recording. On the next night, sleep was polygraphically monitored and an i.v. infusion of saline was given to simulate the experimental condition on the following day.

On the day of the study, the subjects ate breakfast at 0800 and did not consume additional calories by mouth until the end of the study. They were free to drink water and diet decaffeinated beverages. At 1400 , an infusion of $10 \%$ dextrose was started at a constant rate of 5 $\mathrm{g} / \mathrm{kg}$ per $24 \mathrm{~h}$ and was maintained for $57 \mathrm{~h}$. At 1600 , a catheter for blood sampling was placed in the contralateral arm. Blood sampling at 20-min intervals started at 1800 and continued for the next $53 \mathrm{~h}$. The i.v. line was kept patent by a slow drip of heparinized saline. The 4-h delay between the start of glucose infusion and the start of blood collection allowed glucose levels to equilibrate after the transient rise which accompanies the beginning of exogenous infusion (6). The 2-h delay between insertion of the sampling catheter and beginning of sample collection ensured that stress effects related to venipuncture had subsided. During the first night, the subjects slept in total darkness from 2300-0700 and sleep was polygraphically monitored. During sleep times, the catheter was connected to a plastic tubing (length $250 \mathrm{~cm}$, dead space $2 \mathrm{ml}$ ) extending to the adjacent room through a hole in the wall so that sampling could continue without disturbing the subject. Sleep was interrupted at 0700 and the subjects remained awake for the next 28 h, i.e., until 1100 the next morning. Naps were not allowed. Light intensity was 100-200 lux throughout the periods of wakefulness. The subjects remained seated in a comfortable armchair, watched television, read, played board games, and engaged in conversation with staff and visitors. They were allowed to use the bathroom, which was located within $15 \mathrm{ft}$ of the armchair, but otherwise remained seated throughout the waking portions of the study. At 1100 , i.e., $12 \mathrm{~h}$ later than the usual bedtime of 2300 , the subjects went to sleep in complete darkness for an 8-h period. Sleep was polygraphically monitored. Procedures for blood sampling were as during sleep at normal hours. After awakening, blood sampling continued until 2300 when the glucose infusion was tapered off and the subjects were fed and discharged.

Assays. Glucose, C-peptide, insulin, GH, and cortisol were measured in duplicate on each blood sample. For each hormone, all samples from the same subject were analyzed in the same assay.

Plasma glucose was measured using an automatic analyzer (Mode 23A; Yellow Springs Instrument Co., Yellow Springs, $O H$ ) with a coeffcient of variation of $<2 \%$. Plasma C-peptide levels were assayed as described previously (8). The lower limit of the assay is $20 \mathrm{pmol} / \mathrm{liter}$ and the intraassay coefficient of variation averaged $6 \%$. Serum insulin concentrations were determined by a double antibody technique with a lower level of sensitivity of $20 \mathrm{pmol} / \mathrm{liter}$ and an average intraassay coefficient of variation of $6 \%$ (9). GH levels were measured by a modification of a previously described RIA with a lower level of sensitivity of $0.4 \mu \mathrm{g} /$ liter (10). The mean intraassay coefficient of variation is $14 \%$ in the range $0.4-2.0 \mu \mathrm{g} / \mathrm{liter}, 9 \%$ in the range $3-4 \mu \mathrm{g} / \mathrm{liter}$, and $5 \%$ above 5 $\mu \mathrm{g} /$ liter. Plasma cortisol was determined by radioimmunoassay (Diagnostic Products Corp., Los Angeles, CA). The lower limit of sensitivity of this assay is $15 \mathrm{nmol} /$ liter and the intraassay coefficient of variation averages $5 \%$.

Determination of insulin secretory rates. Each subject participated in a separate outpatient study designed to determine his individual parameters of C-peptide disappearance kinetics (11). A bolus intravenous injection of biosynthetic human C-peptide (Eli Lilly and Co., Indianapolis, IN) was administered during somatostatin infusion ( 500 $\mu \mathrm{g} / \mathrm{h}$; Bachem Fine Chemicals, Torrance, CA). Plasma C-peptide levels were measured at frequent intervals over a 3-h period. The kinetic parameters for C-peptide distribution and clearance were determined for each individual according to a two-compartment model using the BMDP 3R program for nonlinear least-squares regression (BMDP Statistical Software, Los Angeles, CA). The mean (士SEM) parameter values were $3988 \pm 427 \mathrm{ml}$ for the volume of distribution, $0.070 \pm 0.013$ $\min ^{-1}$ for $\mathrm{K}_{1}, 0.074 \pm 0.007 \mathrm{~min}^{-1}$ for $\mathrm{K}_{2}$, and $0.065 \pm 0.005 \mathrm{~min}^{-1}$ for $\mathrm{K}_{3}$. These values correspond to a short half-life of $4.1 \pm 0.5 \mathrm{~min}$, a long half-life of $27.5 \pm 2.4 \mathrm{~min}$, and a fraction associated with the short halflife of $0.70 \pm 0.02$. The kinetic parameters obtained in each individual were used to derive, in each 20 -min interval between blood samplings, the ISR from the plasma C-peptide concentrations by deconvolution as previously described (11).

Estimation of insulin clearance. The clearance of secreted insulin was estimated during various portions of the study period as the ratio of the area under the ISR curve and the area under the simultaneously measured serum insulin concentrations as previously described (12).

Sleep recording. The polygraphic sleep records were scored visually at 30-s intervals in stages wake, I, II, III, IV, and rapid eye movement (REM) according to the criteria of Rechtschaften and Kales (13). Sleep onset and morning awakening were defined, respectively, as the times of occurrence of the first and last 30-s intervals scored II, III, IV, or REM. The sleep period (SP) was defined as the interval separating sleep onset from final morning awakening. Sleep latency was defined as the time interval separating lights off and sleep onset. The duration of slow-wave (SW) sleep was defined as the sum of the durations of stages III + IV. Sleep efficiency was calculated as (SP - total duration of wake)/total time in bed.

Analysis of the glucose, insulin, ISR, and cortisol profiles. To quantify the long-term (e.g., diurnal) changes in glucose, insulin, ISR, and cortisol independently of the more rapid, pulsatile variations which are known to affect these variables $(6,14,15)$, a smooth best-fit curve was calculated for each individual profile using the robust, nonlinear regression procedure proposed by Cleveland (16) with a window of $6 \mathrm{~h}$. With this window width, temporal changes which take place over time intervals of $6 \mathrm{~h}$ or more are quantitatively characterized while the contribution of more rapid variations is minimized. In the case of ISR, the contribution of the pulsatile variations to the overall temporal pattern was amplified due to the deconvolution procedure and the Cleveland regression method tended to systematically underestimate the magnitude of the long-term changes. Therefore, another procedure, based on repeated periodogram calculations (17), was used to obtain a smooth best-fit curve. The nadirs and acrophases were defined, respectively, as the times of occurrence of minima and maxima in the best-fit curve. The value of a nadir (acrophase) was defined as the level of the best-fit curve at the nadir (acrophase). The increment associated with an acrophase was defined as the difference between the value of the acrophase and the value of the preceding nadir and was expressed either in measurement units (i.e., concentration or secretion rate) or as a percentage of the value of the preceding nadir (i.e., percent increase over preceding nadir).

Analysis of the GH profiles. Because GH secretion normally occurs as a series of pulses superimposed over basal levels which are around the detection limit of most available assays (15), the calculation of a smooth regression curve as performed for the other profiles is not applicable. Instead, each GH profile was analyzed using a modification of ULTRA, a computer program for pulse detection and quantification (18). The threshold for significance of a pulse was set at three times the intraassay coefficient of variation in the relevant range of concentration. For each significant pulse, the amount of GH secreted during both the ascending and declining limb of the pulse was estimated by integrating the equation $\mathrm{d} C / \mathrm{d} t=\mathrm{S}-\mathrm{kC}$, describing the instantaneous changes of the plasma concentration $\mathrm{C}$ resulting from secretion at rate $\mathrm{S}$ and disappearance at a rate $\mathrm{kC}$, where $\mathrm{k}$ is related to the half-life $t_{1 / 2}$ of the hormone through $\mathrm{k}=\ln 2 / t_{1 / 2}(19)$. This calculation is based on a one-compartment model for $\mathrm{GH}$ clearance. A half-life of $19 \mathrm{~min}$ and a volume of distribution of $7 \%$ of body weight were used in these calcula- 
tions (20). The total amount of $\mathrm{GH}$ secreted over a given time interval was determined by summing the amounts secreted in each of the significant pulses occurring during that time interval.

Statistical analysis. All results are expressed as the mean \pm SEM. Comparisons between parameters measured during nocturnal sleep, sleep deprivation, and daytime sleep are based on an analysis of variance for repeated determinations.

\section{Results}

Profiles of glucose, ISR, and insulin (Figs. 1 and 2, Table I). Overall mean levels of plasma glucose, serum insulin, and insulin secretion rates during continuous glucose infusion at a rate of $5 \mathrm{~g} / \mathrm{kg}$ per $24 \mathrm{~h}$ were, respectively, $5.77 \pm 0.13 \mathrm{mmol} / \mathrm{liter}$, $102.6 \pm 15.1 \mathrm{pmol} / \mathrm{liter}$, and $130 \pm 17 \mathrm{pmol} / \mathrm{min}$.

Despite the fact that glucose was infused at a constant rate throughout the study, plasma glucose levels underwent large fluctuations over the 53-h sampling period. In addition to the rapid oscillations which are known to occur during constant
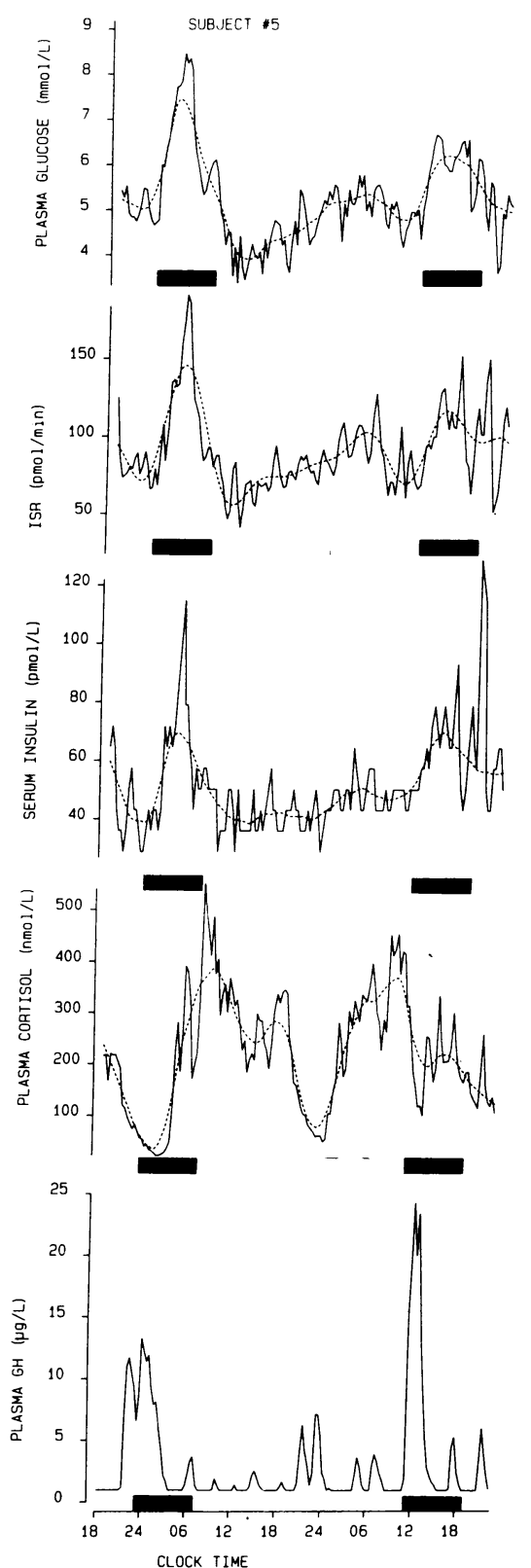

Figure 1. Glucose and hormonal profiles in an individual subject. Profiles of plasma glucose, serum insulin, insulin secretion rates, plasma cortisol, and plasma growth hormone observed in subject 5 during the 53-h study period. Black bars represent sleep periods and the shaded bar represents the period of nocturnal sleep deprivation. The regression curves are shown in dashed lines.
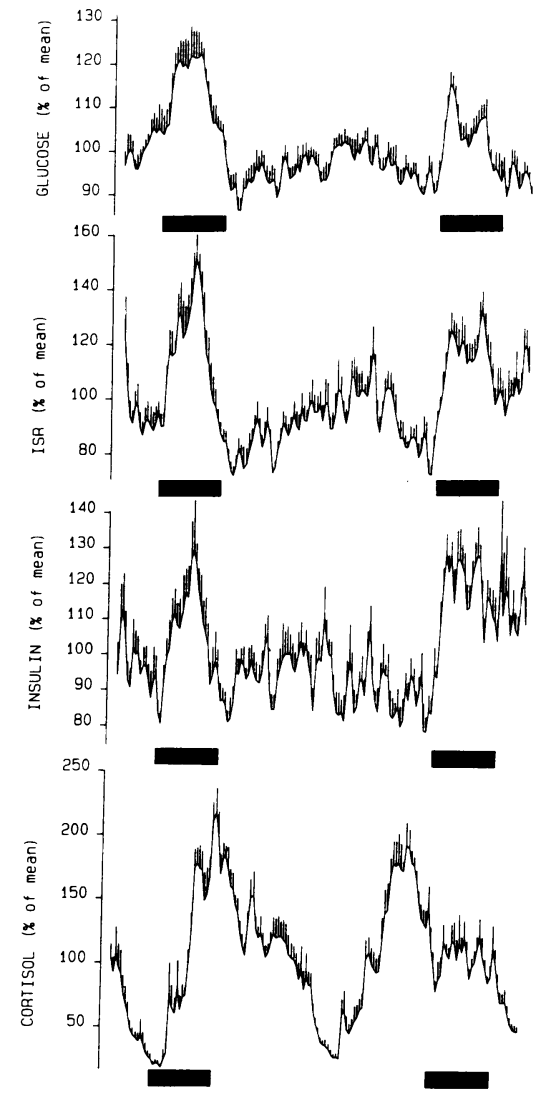

Figure 2. Mean profiles of plasma glucose, serum insulin, insulin secretion rates, cortisol, and growth hormone concentrations for the group of eight subjects. The vertical bars represent SEM. To eliminate the effects of interindividual variations in mean glucose, insulin, ISR, and cortisol level on the group pattern, the individual values were expressed as percentages of the mean. The black bars represent the sleep period. The shaded bar represents the period of nocturnal sleep deprivation.

glucose infusion (6), a consistent pattern with three well-defined acrophases, one during nocturnal sleep, one during sleep deprivation, and one during daytime sleep, was identified by regression analysis. Representative profiles (subject 5) are shown in Fig. 1. The profiles of ISR and serum insulin roughly paralleled the changes in glucose levels. The mean profiles of glucose, ISR, and insulin presented in Fig. 2 clearly demonstrate that, in the face of constant glucose infusion, glucose levels and insulin secretion rose during nocturnal sleep, decreased to rise again and peak during the night of sleep deprivation and, after the resumption of lower daytime levels, increased sharply when sleep occurred during the daytime. Acrophases of insulin concentration were not consistently observed during sleep deprivation. The parameters derived from the regression curve allowed these effects of time of day and sleep to be quantified (Table I).

Glucose levels started rising in the evening and reached an acrophase 3-5 h after sleep onset. This nocturnal acrophase corresponded to an increase of 30\% over late afternoon levels. In the absence of sleep, a nocturnal acrophase nevertheless 
Table I. Quantitative Characteristics of Glucose, ISR, and Insulin Acrophases during Nocturnal Sleep (NS), Nocturnal Wakefulness $(N W)$, and Daytime Sleep (DS; Mean \pm SEM)

\begin{tabular}{|c|c|c|c|c|}
\hline & $\begin{array}{l}\text { Nocturnal } \\
\text { sleep }\end{array}$ & $\begin{array}{c}\text { Nocturnal } \\
\text { wakefulness }\end{array}$ & $\begin{array}{l}\text { Daytime } \\
\text { sleep }\end{array}$ & $\begin{array}{l}\text { Significant } \\
\text { contrasts }\end{array}$ \\
\hline \multicolumn{5}{|l|}{ Glucose } \\
\hline Timing & $02: 35 \pm 33 \mathrm{~min}$ & $02: 13 \pm 50 \mathrm{~min}^{\ddagger}$ & $13: 28 \pm 31 \mathrm{~min}$ & - \\
\hline Max level & $6.99 \pm 0.22 \mathrm{mmol} /$ liter & $6.05 \pm 0.22 \mathrm{mmol} /$ liter $^{\ddagger}$ & $6.16 \pm 0.11 \mathrm{mmol} / \mathrm{liter}$ & $\begin{array}{l}\mathrm{NW}<\mathrm{NS} \\
\mathrm{DS}<\mathrm{NS}\end{array}$ \\
\hline Absolute increase & $1.61 \pm 0.28 \mathrm{mmol} / \mathrm{liter}$ & $0.78 \pm 0.22 \mathrm{mmol} /$ liter $^{+}$ & $0.83 \pm 0.17 \mathrm{mmol} / \mathrm{liter}$ & $\begin{array}{l}\mathrm{NW}<\mathrm{NS} \\
\mathrm{DS}<\mathrm{NS}\end{array}$ \\
\hline Relative increase & $30.6 \pm 5.2 \%$ & $16.6 \pm 4.7 \% *$ & $16.3 \pm 3.4 \%$ & $\begin{array}{l}\mathrm{NW}<\mathrm{NS} \\
\mathrm{DS}<\mathrm{NS}\end{array}$ \\
\hline \multicolumn{5}{|l|}{ ISR } \\
\hline Timing & $02: 58 \pm 13 \mathrm{~min}$ & $00: 23 \pm 72 \mathrm{~min}$ & $13: 48 \pm 10 \mathrm{~min}$ & - \\
\hline Max level & $177 \pm 22 \mathrm{pmol} / \mathrm{min}$ & $148 \pm 20 \mathrm{pmol} / \mathrm{min}$ & $163 \pm 21 \mathrm{pmol} / \mathrm{min}$ & $\mathrm{NW}<\mathrm{NS}$ \\
\hline Absolute increase & $66 \pm 11 \mathrm{pmol} / \mathrm{min}$ & $47 \pm 8 \mathrm{pmol} / \mathrm{min}$ & $53 \pm 5 \mathrm{pmol} / \mathrm{min}$ & ns \\
\hline Relative increase & $60.0 \pm 10.7 \%$ & $49.3 \pm 7.6 \%$ & $54.6 \pm 7.1 \%$ & ns \\
\hline \multicolumn{5}{|l|}{ Insulin } \\
\hline Timing & $02: 45 \pm 50 \mathrm{~min}$ & - & $14: 35 \pm 24 \mathrm{~min}$ & - \\
\hline Max level & $116 \pm 14 \mathrm{pmol} / \mathrm{liter}$ & - & $124 \pm 17 \mathrm{pmol} /$ liter & ns \\
\hline Absolute increase & $32 \pm 6 \mathrm{pmol} / \mathrm{liter}$ & - & $35 \pm 6 \mathrm{pmol} / \mathrm{liter}$ & ns \\
\hline Relative increase & $41.1 \pm 9.7 \%$ & - & $38.6 \pm 4.8 \%$ & ns \\
\hline
\end{tabular}

${ }^{*} P<0.05$ at least. ${ }^{\ddagger} n=7$, one of the subjects had no glucose acrophase during NW.

occurred in seven of the eight subjects, suggesting the existence of an intrinsic circadian variation in glucose regulation. The magnitude of this circadian rise was roughly half the amplitude of the rise seen during nocturnal sleep (Table I). Morning glucose levels after a night of sleep deprivation were similar to those observed on the preceding day after a night of sleep. Indeed, mean glucose levels during the time interval 0800-1100 were $5.27 \pm 0.24 \mathrm{mmol} / \mathrm{liter}$ after the night of sleep and $5.33 \pm 0.16 \mathrm{mmol} / \mathrm{liter}$ after the night of sleep deprivation. When the subjects went to sleep in the morning, glucose levels peaked on average $2 \frac{1}{2} \mathrm{~h}$ later and this sleep-associated rise represented an elevation of $16.3 \pm 3.4 \%$ above presleep levels (Table I). The comparison of the respective sizes of the circadian elevation in the absence of sleep and of the sleep elevation at an abnormal circadian time suggests that, in normal conditions of nocturnal sleep, the effects of sleep and time of day are superimposed.

Analysis of the ISR profiles revealed that well-defined acrophases occurred in all subjects during nocturnal sleep, during nocturnal wakefulness, and during daytime sleep. The timing of these ISR acrophases did not differ significantly from the timing of the corresponding glucose acrophases. When expressed as percentage increase over basal level, the elevations in ISR, whether associated with nocturnal sleep, nocturnal wakefulness, or daytime sleep, were of significantly larger magnitude than those observed for glucose $(P<0.03$ at least). In contrast to what was observed for glucose, elevations of ISR during nocturnal wakefulness and during daytime sleep were not of lesser magnitude than those seen during nocturnal sleep (Table I).
Profiles of insulin concentrations paralleled those of glucose and ISR during nocturnal sleep and during daytime sleep but a nocturnal acrophase in the absence of sleep was detected in only four of the eight subjects. Thus, a maximum of insulin concentrations during sleep deprivation is not apparent in the mean profile of insulin concentrations (Fig. 2). This lack of parallelism between the profiles of insulin secretion and peripheral insulin concentrations during sleep deprivation reflected a circadian variation in insulin clearance, with clearance rates $30-40 \%$ higher during the first half of the habitual sleep period $(2300-0300 ; 1.57 \pm 0.20 \mathrm{liter} / \mathrm{min})$ than in the morning $(0800$ $1100 ; 1.16 \pm 0.13 \mathrm{liter} / \mathrm{min}, P<0.01)$. During nocturnal and daytime sleep, the acrophases of insulin concentrations occurred at approximately the same time as the corresponding glucose acrophases. During nocturnal sleep, the magnitude of the insulin acrophase was intermediate between that of glucose and that of ISR, without being significantly different from either one. During daytime sleep, the magnitude of the insulin acrophase was significantly larger than that of glucose, but did not differ significantly from that of ISR. Insulin clearance during nocturnal sleep $(2300-0300 ; 1.64 \pm 0.21 \mathrm{liter} / \mathrm{min})$ was $>40 \%$ higher than on the following morning (0800-1100; $1.16 \pm 0.13 \mathrm{liter} / \mathrm{min}, P<0.01)$. However, during daytime sleep, insulin clearance was intermediate between the morning and nighttime value, without differing significantly from either one (1100-1500; 1.33 $\pm 0.13 \mathrm{liter} / \mathrm{min})$.

Cortisol and GH profiles during nocturnal sleep and nocturnal wakefulness (Figs. 1 and 2, Table II). The cortisol profile of subject 5 is shown, as an example, in Fig. 1 and the mean cortisol profile for the group of eight subjects is illustrated in 
Fig. 2. The nocturnal rise of cortisol started 1-2 $h$ after sleep onset when sleep occurred at its normal time, and a morning maximum was observed within $1 \mathrm{~h}$ after morning awakening. Cortisol levels then declined throughout the day to reach a late evening nadir before the usual bedtime. Despite the absence of sleep, and the persistence of constant conditions of light and activity, cortisol levels rose again during the night and reached a maximum at approximately the same clock time as after the night with normal sleep. Table II gives the mean values for the parameters quantifying the circadian variation of cortisol during nocturnal sleep and nocturnal wakefulness. The evening to morning excursion was of smaller amplitude during sleep deprivation than under normal conditions of nocturnal sleep and the evening nadir tended to occur earlier. The diminished circadian amplitude of cortisol levels during sleep deprivation was related to the occurrence of a slightly higher nadir as well as of a lower morning acrophase (Table II).

The profile of plasma $\mathrm{GH}$ concentrations observed in subject 5 during the 53-h study period is shown in Fig. 1 and the mean GH profiles for the group are illustrated in Fig. 2. Variations in GH levels consisted of a series of pulses occurring over stable baseline levels which were around the sensitivity of the assay. These GH profiles observed during constant glucose infusion were similar in every respect to previously reported profiles of GH secretion in healthy nonobese male adults studied during normal feeding conditions (15). During the baseline night, significant GH pulses occurred during the hour preceding sleep onset in five of the eight subjects, and after sleep onset in all subjects. Comparisons of nocturnal GH secretion in the presence and in the absence of sleep were therefore based on the time interval 2200-0200. In the absence of sleep, significant pulses of $\mathrm{GH}$ were detected between 2200 and 0200 in five of the eight subjects. However, these nocturnal GH pulses which occurred during wakefulness were of smaller amplitude than those observed during normal sleep. As indicated in Table

Table II. Quantitative Characteristics of Plasma Cortisol and Growth Hormone Profiles during Nocturnal Sleep and Nocturnal Wakefulness (Mean \pm SEM)

\begin{tabular}{|c|c|c|c|}
\hline & $\begin{array}{l}\text { Nocturnal } \\
\text { sleep }\end{array}$ & $\begin{array}{c}\text { Nocturnal } \\
\text { wakefulness }\end{array}$ & $\begin{array}{l}P \text { level of } \\
\text { difference }\end{array}$ \\
\hline Cortisol & & & \\
\hline $\begin{array}{l}\text { Timing of nadir } \\
\text { Level of nadir }\end{array}$ & $23: 50 \pm 17 \mathrm{~min}$ & $22: 58 \pm 19 \mathrm{~min}$ & $P<0.09$ \\
\hline $\begin{array}{l}(\mathrm{nmol} / \mathrm{liter}) \\
\text { Timing of acrophase }\end{array}$ & $\begin{array}{c}52.1 \pm 10.8 \\
07: 30 \pm 19 \mathrm{~min}\end{array}$ & $\begin{array}{c}70.1 \pm 6.6 \\
08: 03 \pm 21 \mathrm{~min}\end{array}$ & $\begin{array}{l}\text { ns } \\
\text { ns }\end{array}$ \\
\hline $\begin{array}{l}\text { Level of acrophase } \\
\text { (nmol/liter) }\end{array}$ & $372.5 \pm 22.1$ & $357.6 \pm 23.2$ & $P<0.04$ \\
\hline $\begin{array}{l}\text { Absolute increment } \\
\text { (nmol/liter) }\end{array}$ & $320.3 \pm 21.0$ & $287.8 \pm 22.9$ & $P<0.04$ \\
\hline Growth hormone & & & \\
\hline $\begin{array}{l}\text { Number of pulses } \\
\text { between } 2200 \text { and } \\
0200\end{array}$ & $2.0 \pm 0.4$ & $1.0 \pm 0.4$ & $P=0.10$ \\
\hline $\begin{array}{l}\text { Amount secreted } \\
\text { between } 2200 \text { and } \\
0200(\mu g)\end{array}$ & $403 \pm 82$ & $119 \pm 48$ & $P<0.04$ \\
\hline
\end{tabular}

Table III. Quantitative Characteristics of Sleep and Sleep-related Growth Hormone Secretion during Nocturnal Sleep and Daytime Sleep (Mean \pm SEM)

\begin{tabular}{lccc}
\hline & $\begin{array}{c}\text { Nocturnal } \\
\text { sleep }\end{array}$ & $\begin{array}{c}\text { Daytime } \\
\text { sleep }\end{array}$ & $\begin{array}{c}P \text { level of } \\
\text { difference }\end{array}$ \\
\hline Sleep period & $440 \pm 11 \mathrm{~min}$ & $411 \pm 13 \mathrm{~min}$ & $\mathrm{~ns}$ \\
Sleep efficiency & $86.7 \pm 2.6 \%$ & $85.7 \pm 4.1 \%$ & $\mathrm{~ns}$ \\
Sleep latency & $25 \pm 6 \mathrm{~min}$ & $8 \pm 1 \mathrm{~min}$ & $P<0.02$ \\
Amount of SW & $69 \pm 10 \mathrm{~min}$ & $101 \pm 9 \mathrm{~min}$ & $P<0.002$ \\
Amount of REM & $80 \pm 9 \mathrm{~min}$ & $57 \pm 13 \mathrm{~min}$ & $P<0.02$ \\
Amount of wake & $48 \pm 10 \mathrm{~min}$ & $61 \pm 17 \mathrm{~min}$ & $\mathrm{~ns}$ \\
$\begin{array}{l}\text { Amount of SW in first } \\
\quad \text { 2 h of sleep }\end{array}$ & $34 \pm 5 \mathrm{~min}$ & $48 \pm 4 \mathrm{~min}$ & $P<0.02$ \\
$\begin{array}{l}\text { Amount of GH secreted } \\
\text { in first 2 h of sleep }\end{array}$ & $293 \pm 90 \mu \mathrm{g}$ & $305 \pm 75 \mu \mathrm{g}$ & $\mathrm{ns}$ \\
\hline
\end{tabular}

II, the amount of GH secreted in the time interval 2200-0200 in the absence of sleep was on average less than one-third of the amount secreted in the presence of sleep.

Sleep and effects of sleep onset (Fig. 3, Table III). The characteristics of nocturnal and daytime sleep are presented in Table III. Sleep duration and efficiency were similar under both study conditions. When sleep was allowed during the daytime after $28 \mathrm{~h}$ of continuous wakefulness, sleep latency was significantly shortened as compared to baseline conditions and the amount of SW stage was increased by $\sim 50 \%$. In contrast, the total duration of REM stages was significantly reduced.

Fig. 3 illustrates the effects of sleep onset on mean glucose, ISR, cortisol, and GH levels for both nocturnal sleep and daytime sleep. Before calculating these mean profiles, the individual data were referenced to the timing of the first sample collected after sleep onset (denoted $0 \mathrm{~min}$ ) and, for glucose, ISR, and cortisol, expressed as a percentage of the overall mean level. The significance of effects of sleep onset on glucose, ISR, and cortisol profiles (indicated on Fig. 3) was determined by comparing the mean level during the 2 -h period preceding and following the timing of the first sample collected after sleep onset.

After nocturnal sleep onset, levels of plasma glucose, insulin secretion, and plasma insulin (not shown) rose significantly above presleep values. These sleep-associated rises tended to be more pronounced when sleep onset occurred during the daytime, but the differences failed to reach significance. Before daytime sleep, presleep glucose and ISR levels tended to be lower than before nighttime sleep $(P=0.10$ for glucose, $P$ $=0.07$ for ISR) and maximum levels after daytime sleep onset were observed within $2-3 \mathrm{~h}$, in contrast to 3-4 h for nocturnal sleep onset (Table I, Fig. 3).

Before nocturnal sleep, cortisol levels were low and were not significantly affected by sleep onset. However, after onset of sleep in the late morning, a marked inhibition of cortisol secretion was apparent, with a drop in mean cortisol level averaging $48.5 \pm 10.9 \%$ (Fig. 3). As expected, sleep onset reproducibly stimulated $\mathrm{GH}$ secretion and a significant $\mathrm{GH}$ pulse started within $20 \mathrm{~min}$ after sleep onset in all subjects when sleep occurred at the normal nighttime and in seven of the eight subjects when sleep occurred in the morning. As shown in Table III, sleep onset GH secretion was of similar magnitude when sleep started in the late evening or in the late morning despite 


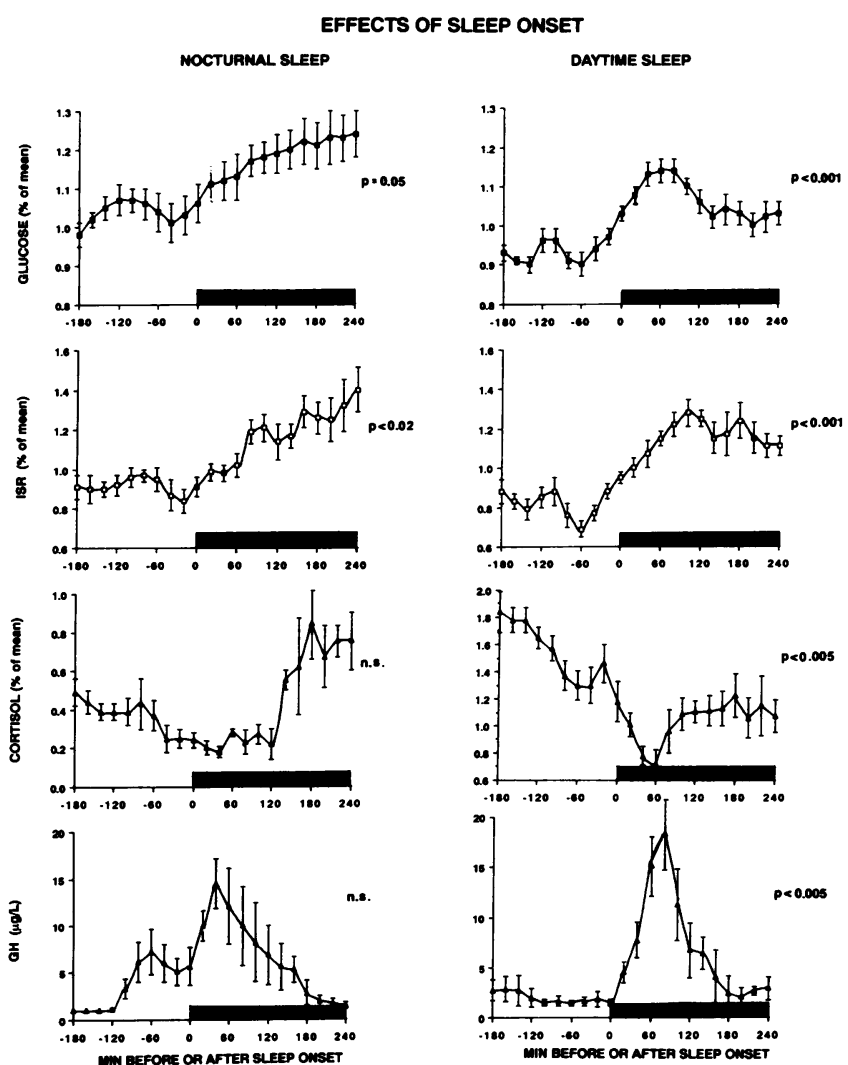

Figure 3. Effects of sleep onset. Mean ( \pm SEM) glucose levels, insulin secretion rates, cortisol, and growth hormone concentrations before and after nocturnal sleep onset (left) and daytime sleep onset following a period of $28 \mathrm{~h}$ of sleep deprivation (right). Time 0 on the abscissa corresponds to the time of collection of the first sample following sleep onset as defined by the polygraphic recordings. For glucose, ISR, and cortisol, the data for each subject were expressed as a percentage of his overall mean level to take into account interindividual variations in mean levels.

the fact that more SW stages occurred in early sleep in the morning.

Relationships between patterns of glucose and ISR and patterns of cortisol and GH (Figs. 4-6). Correlation matrices for all quantitative parameters characterizing the circadian and sleeprelated changes in glucose levels, ISR, cortisol concentrations, and GH secretion were calculated to identify significant associations between patterns of glucose and/or ISR, on the one hand, and patterns of counterregulatory hormone secretion, on the other hand. Multiple regression analyses were performed wherever appropriate, but in no case resulted in the identification of more than one significant independent variable. These analyses were conducted separately for the period of sleep deprivation and for the periods of nocturnal and daytime sleep.

During the period of sleep deprivation, there were no signifcant correlations between parameters quantifying $\mathrm{GH}$ secretion and parameters quantifying the nocturnal rises of glucose and ISR. Examination of the mean profiles of ISR and cortisol (Fig. 4) suggested the existence of an inverse relationship between temporal changes in pancreatic insulin secretion and circulating cortisol levels. Indeed, ISR was minimal in the early morning, when cortisol levels were at their peak, and increased throughout the day, while cortisol concentrations continuously

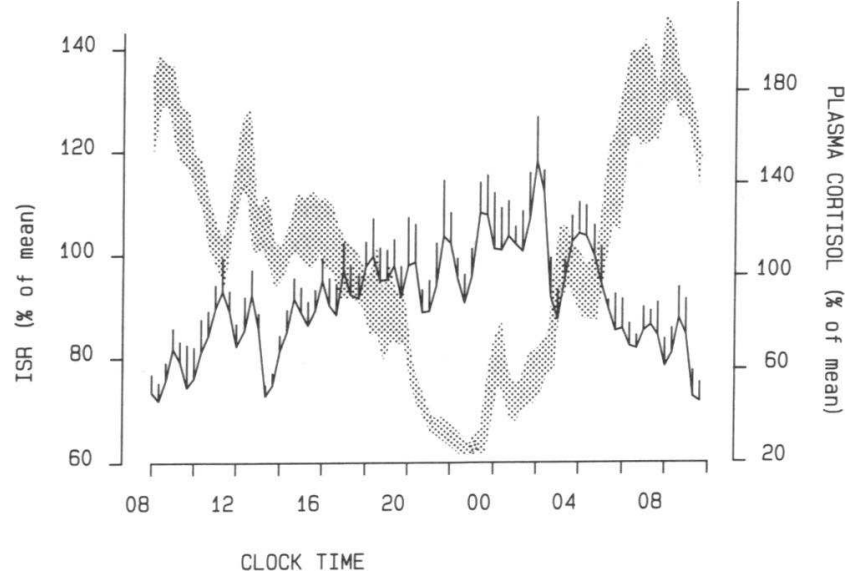

Figure 4. Relationship between the profile of insulin secretion rates and the cortisol rhythm during sleep deprivation. Mean profiles of insulin secretion rate (ISR; solid lines, SEM shown as vertical line at each time point) and cortisol (shaded area corresponds to mean \pm SEM) during the period of continuous wakefulness without recumbency from 0800 to 1000 the next day. Note the remarkable mirror-like relationship between the variations of ISR and those of cortisol. When expressed as percentage of the mean level, the magnitude of the rise in ISR tended to correlate with the size of the decline in cortisol concentration $(r=0.62, n=8, P<0.10)$.

declined. The nadir of cortisol generally preceded the nocturnal ISR maximum. In the latter part of the night of sleep deprivation and during the following morning, ISR declined while cortisol concentrations rose. Fig. 5 shows that the amplitudes of the diurnal variations of cortisol and ISR, when expressed as a percentage of the mean level, were positively correlated. Thus, the larger the cortisol drop from morning acrophase to evening nadir, the higher the ISR rise from morning nadir to nocturnal acrophase. This correlation between diurnal amplitudes of ISR and cortisol was found both during conditions of normal sleep $(r=0.67, P=0.07, n=8)$ and during sleep deprivation $(r=0.62, P<0.10, n=8)$ and was statistically significant when both conditions were considered together $(r$ $=0.67, P<0.005, n=16$ ). Correlations between amplitude of the diurnal glucose variations and amplitude of the cortisol rhythm were not significant $(r=0.25, P>0.30, n=16)$.

The increases in glucose and ISR after sleep onset did not correlate with changes in cortisol levels for either nocturnal or

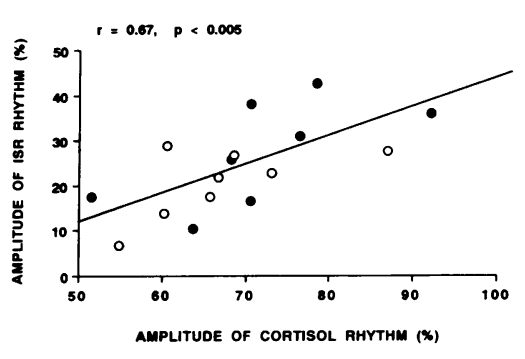

Figure 5. Relationship between the amplitude of the diurnal variation in insulin secretion and the amplitude of the cortisol rhythm. Correlation between the relative amplitude of the insulin secretion rate rhythm $(50 \%$ of difference between acrophase and nadir, divided by mean ISR level) and the relative amplitude of the cortisol rhythm ( $50 \%$ of difference between acrophase and nadir, divided by mean cortisol level) during the study period with nocturnal sleep (closed circles) and during the study period with sleep deprivation (open circles). 
daytime sleep. However, during nocturnal sleep, the absolute increase in glucose levels was strongly correlated with the amount of GH secreted between 2200 and $0200(r=0.84, P$ $<0.01, n=8$; Fig. 6 ). A similar positive association between the magnitude of the glucose elevation during sleep and the amount of GH secreted was observed during daytime sleep but failed to reach statistical significance $(r=0.56, P=0.15, n=8$, Fig. 6). When nocturnal and daytime sleep were considered together, the coefficient of correlation between sleep-related glucose elevation and amount of $\mathrm{GH}$ secreted was 0.73 ( $P$ $<0.002, n=16$, Fig. 6). Correlations between sleep-related increases in ISR levels and amount of GH secreted failed to reach significance.

\section{Discussion}

In this study, glucose levels and insulin secretion were continuously monitored during constant glucose infusion for a total of $53 \mathrm{~h}$, including an 8-h period of nocturnal sleep, a 28 -h period of sleep deprivation, and an 8-h period of daytime sleep. The profiles observed under these conditions indicate unequivocally that both circadian rhythmicity and sleep modulate glucose regulation. Indeed, during sleep deprivation, glucose concentrations and insulin secretion increased steadily to reach a nocturnal maximum at approximately the same time as during normal sleep and then returned to daytime levels. After sleep onset in the late morning, a sharp rise in glucose levels and insulin secretion was again observed. The quantitative analysis of the size of the elevations seen in the absence of sleep and during daytime sleep suggests that, in normal conditions of nocturnal sleep, both effects are superimposed. Not unexpectedly, in individual profiles, and particularly in the case of the profiles of insulin secretion, the sizes of the circadian and sleeprelated elevations did not usually add up arithmetically to the size of the normal nocturnal rise. Indeed, the size of the nocturnal rise seen during the first night of sleep was probably underestimated because the study was started at 1800 , with levels of glucose and ISR already above than morning values. Moreover, the characteristics of daytime recovery sleep after $28 \mathrm{~h}$ of uninterrupted wakefulness differed significantly from those of baseline sleep.

The experimental protocol which allowed these effects of circadian rhythmicity and sleep to be demonstrated took advantage of the fact that circadian rhythms take several days to adapt to a change of sleep-wake and light-dark cycles (15). Thus, by abruptly delaying the sleep period by $12 \mathrm{~h}$, the effects of time of day could be observed in the absence of sleep, and the

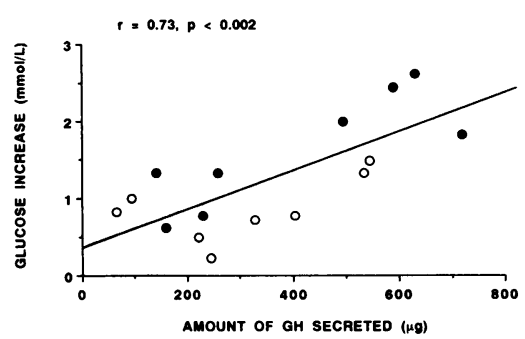

Figure 6. Relationship between glucose elevations during sleep and concomitant growth hormone secretion. Correlation between glucose increase during nocturnal sleep (closed circles) and during daytime sleep (open circles) and amount of GH se-

creted during the time interval 2200-0200 (for nocturnal sleep) or 1000-1400 (for daytime sleep). effects of sleep could be observed at an abnormal circadian time, i.e., a time when sleep does not normally occur. To optimize interindividual synchronization, the subjects complied with a standardized schedule of sleep for one week before the study. An extended period of habituation served to minimize possible stress effects of hospitalization, laboratory procedures, and novel social environment. Confounding effects of food ingestion and prolonged fasting were avoided by replacing the normal caloric intake by a constant glucose infusion, thereby creating a steady-state condition with levels of glucose and insulin secretion within the physiologic range. During wakefulness, the level of physical activity was minimal and essentially constant. Sleep onset and maintenance were polygraphically monitored.

The profiles of peripheral glucose and insulin concentrations and of insulin secretion rates observed under these experimental conditions confirmed and extended the findings of previous studies showing decreased glucose tolerance in the afternoon and evening as compared to the morning (1-5). The decrease in glucose tolerance continued throughout the evening and early part of the habitual sleep period. Irrespective of the sleep or wake condition, glucose levels and insulin secretion rates returned spontaneously to basal morning values. The magnitude of the nocturnal rise in the absence of sleep averaged $17 \%$ for glucose and almost $50 \%$ for insulin secretion. This marked increase in insulin secretion was not associated with a significant nocturnal elevation of serum insulin concentrations. This finding indicates the existence of a diurnal variation in insulin clearance, consisting of a nocturnal increase concomitant and almost commensurate with the rise in insulin secretion. Direct, independent measurements of insulin clearance at different times of day will be needed to confirm this observation. Reduced sensitivity of peripheral tissues to insulin has been shown to be a major cause of decreased glucose tolerance in the evening as compared to the morning (4) but inappropriate low insulin secretion has been implicated as well $(1,2,5)$. In this study, the simultaneous measurement of plasma levels of insulin and C-peptide, and the subsequent derivation of insulin secretion rates based on individual kinetic parameters for Cpeptide clearance and metabolism, showed that the effects of decreased insulin sensitivity and insufficient insulin secretion are compounded by a marked acceleration of the disposal of secreted insulin.

The persistence of the diurnal variation of glucose tolerance in the absence of sleep and in the face of constant environmental conditions, continuously low levels of activity, and constant caloric intake strongly suggests that this diurnal variation must be, at least partially, controlled by signals originating from a robust circadian pacemaker. In mammalian species, increasing evidence points at the existence of a single central pacemaker, located in the suprachiasmatic nuclei of the hypothalamus, as the mechanism responsible for circadian rhythmicity (21). So far, hormonal signalling appears to be the primary pathway for the transmission of centrally generated circadian oscillations to peripheral organs. Modulatory effects of circadian rhythmicity on glucose control could be mediated by counterregulatory hormones. Current concepts of glucose counterregulation in humans are primarily based on extensive studies of hypoglycemia, where abnormally low glucose levels are associated with increased secretion of epinephrine, glucagon, growth hormone, and cortisol. Recent studies have also shown that prolonged fasting is associated with large increases in both $\mathrm{GH}$ and corti- 
sol secretion $(22,23)$. Our study raises the possibility that rises in counterregulatory hormone concentrations that are not directly caused by metabolic conditions may modulate glucose regulation under physiologic conditions. Indeed, during the 28-h period of sleep deprivation, we observed an inverse temporal relationship between the patterns of glucose and insulin secretion, on the one hand, and that of plasma cortisol, on the other hand. Furthermore, the amplitude of the diurnal variation in insulin secretion was significantly correlated with the amplitude of the cortisol rhythm. Studies examining the patterns of glucose and insulin secretion following experimentally induced modifications of the cortisol rhythm will be needed to confirm this correlative evidence suggesting a role for cortisol in controlling the diurnal variation in glucose tolerance under normal physiologic conditions. Among the other counterregulatory hormones, only epinephrine is modulated by circadian rhythmicity but the amplitude of the rhythm is less than half that of cortisol and the waveshape of the pattern is neither parallel to, nor in a mirror-image relationship with, that observed for glucose tolerance (24). Glucagon and GH concentrations do not undergo consistent diurnal variations $(6,15)$.

This study demonstrated that sleep onset, irrespective of the time of day when it occurs, has profound effects on glucose regulation. Indeed, sleep onset resulted in a $10-20 \%$ elevation of plasma glucose, a $50-60 \%$ increase in insulin secretion rates, and a $35-45 \%$ rise in serum insulin. Significant effects of sleep onset on insulin clearance could not be demonstrated. The effects of sleep onset per se tended to be more pronounced after daytime sleep after $28 \mathrm{~h}$ of continuous wakefulness than when sleep occurred at the usual bedtime after a normal 16-h period of daytime wakefulness. As expected, sleep onset was also associated with a large pulse of GH secretion. After nocturnal sleep, the absolute rise in glucose levels was strongly correlated with the amount of GH secretion and cortisol levels were not affected by sleep onset. After daytime sleep, there was a weaker association between the magnitude of the glucose rise and the amount of GH secreted and, in accordance with classical findings (25), cortisol levels abruptly declined by $50 \%$.

Little is known about the set-point of glucose regulation during sleep. Normal subjects studied during an overnight fast generally show stable or slightly declining levels of glucose and insulin (26-28). However, we are unaware of a single study where the onset and maintenance of sleep were polygraphically monitored and where the experimental conditions allowed for continuous blood sampling for the measurement of glucose, insulin, and counterregulatory hormone levels without disturbing or interrupting the subject's sleep. Thus, while the differences in the nocturnal patterns of glucose and insulin seen in this study and those seen during overnight fasting are likely to reflect primarily the effects of constant glucose infusion versus prolonged fasting, it must be recognized that, during normal overnight fasting, patterns of glucose tolerance in the presence of sleep and of sleep-related changes in counterregulatory hormones have not yet been defined. In a recent study, Clore et al. (28) have reported that, in normal fasted men kept awake throughout the night while at bed rest, both glucose production and glucose utilization decrease by $14 \%$ on average, while in subjects who are permitted to sleep, these decreases were 20$30 \%$. This study thus indicated the existence of a reduction of both glucose production and glucose utilization during sleep (28). The size of the reduction in glucose utilization, i.e., $\sim 10 \%$, is consistent with the reduction in muscular activity during sleep. In our study, however, the rises in glucose levels observed during daytime sleep, i.e., in the absence of superimposed effects of circadian rhythmicity, were well in excess of $10 \%$ and should thus involve either larger decreases in glucose utilization or increased hepatic glucose output. It is now established that physiological elevations of GH levels can cause a rapid decrease in glucose-induced glucose uptake and simultaneously augment endogenous glucose production $(29,30)$. Pulsatile administration of bovine $\mathrm{GH}$ in dogs induces a rapid increase in hepatic glucose output (31). The correlations between the magnitude of the sleep-associated glucose rise and the concomitant amount of GH secretion seen in this study suggest that the effects of sleep on glucose tolerance may be partially mediated by the increase in GH secretion associated with sleep onset. Further studies will be necessary to delineate the respective roles of glucose production and glucose utilization in the sleep-associated changes in the set point of glucose regulation.

In conclusion, our study has demonstrated that both circadian rhythmicity and sleep are important physiological regulators of glucose tolerance in normal man. These findings suggest that time of day may be important for the diagnosis and treatment of conditions of impaired glucose tolerance.

\section{Acknowledgments}

We are indebted to the volunteers who agreed to participate in these demanding studies. We thank Mrs. Diane Nummy for expert completion of the sleep studies and the nursing staff of the General Clinical Research Center at the University of Chicago for skillful assistance.

This work was supported by grants DK-41814, DK-20595, and DK-31842 from the National Institutes of Health and the General Clinical Research Center at the University of Chicago.

\section{References}

1. Carroll, K., and P. Nestel. 1973. Diurnal variation in glucose tolerance and in insulin secretion in man. Diabetes. 22:333-348.

2. Jarrett, R. J. 1979. Rhythms in insulin and glucose. In Endocrine Rhythms. D. T. Krieger, editor. Raven Press Ltd., New York. 247-258.

3. Service, F. J., L. D. Hall, R. E. Westland, P. C. O'Brien, V. L. W. Go, M. W. Haymond, and R. A. Rizza. 1983. Effects of size, time of day and sequence of meal ingestion on carbohydrate tolerance in normal subjects. Diabetologia. 25:316-321

4. Verrillo, A., A. De Teresa, C. Martino, G. Di Chiara, M. Pinto, L. Verrillo, F. Torello, and A. Gattoni. 1989. Differential roles of splanchic and peripheral tissues in determining diurnal fluctuation of glucose tolerance. Am. J. Physiol. 257:E459-E465.

5. Lee, A., T. Agajanian, and G. A. Bray. 1985. Diurnal variation of insulin sensitivity exists in normal subjects but not in obese subjects. Diabetes. 34:125a.

6. Shapiro, E. T., H. Tillil, K. S. Polonsky, V. S. Fang A. H. Rubenstein, and E. Van Cauter. 1988. Oscillations in insulin secretion during constant glucose infusion in normal man: relationship to changes in plasma glucose. J. Clin. Endocrinol. Metab. 67:307-314.

7. Van Cauter, E., D. Desir, C. Decoster, F. Féry, and E. O. Balasse. 1989 Nocturnal decrease of glucose tolerance during constant glucose infusion. J. Clin. Endocrinol. Metab. 69:604-611.

8. Faber, O. K., C. Binder, J. Markussen, L. G. Heding, V. K. Naithani, H. Kuzuya, P. Blix, D. L. Horwitz, and A. H. Rubenstein. 1978. Characterization of seven C-peptide antisera. Diabetes. 27:170-177.

9. Morgan, C., and A. Lazarow. 1963. Immunoassay of insulin: two antibody systems: plasma insulin levels of normal, subdiabetic and diabetic rats. Diabetes. 12:115-126.

10. Virasoro, E., G. Copinschi, O. D. Bruno, and R. Leclercq. 1971. Radioimmunoassay of human growth hormone using a charcoal-dextran separation procedure. Clin. Chim. Acta. 31:294-297.

11. Polonsky, K. S., J. Licinio-Paixao, B. D. Given, W. Pugh, P. Rue, J. Galloway, T. Karrison, and B. Frank. 1986. Use of biosynthetic human C-peptide in the measurement of insulin secretion rates in normal volunteers and type $I$ diabetic patients. J. Clin. Invest. 77:98-105. 
12. Shapiro, E. T., H. Tillil, M. A. Miller, B. H. Frank, J. A. Galloway, A. H. Rubenstein, and K. S. Polonsky. 1987. Insulin secretion and clearance: comparison after oral and intravenous glucose. Diabetes. 36:1365-1371.

13. Rechtschaffen, A., and A. Kales. 1968. A manual of standardized terminology, techniques and scoring system for sleep stages of human subjects. U.S. Government Printing Office, Washington, DC.

14. Simon, C., G. Brandenberger, and M. Follenius. 1987. Ultradian oscillations of plasma glucose, insulin, and C-peptide in man during continuous entera nutrition. J. Clin. Endocrinol. Metab. 64:669-674.

15. Van Cauter, E., and J. Aschoff. 1989. Endocrine and other biologica rhythms. Endocrinology. L. J. DeGroote, editor. W. B. Saunders Co., Philadelphia. 2658-2705.

16. Cleveland, W. 1979. Robust locally weighted regression and smoothing scatterplots. J. Am. Stat. Assoc. 74:829-836.

17. Van Cauter, E. 1979. Method for characterization of 24-h temporal variation of blood constituents. Am. J. Physiol. 237:E255-E264.

18. Van Cauter, E. 1988. Estimating false-positive and false-negative errors in analyses of hormonal pulsatility. Am. J. Physiol. 254:E786-E794.

19. Golstein, J., E. Van Cauter, D. Desir, P. Noel, J. Spire, S. Refetoff, and G. Copinschi. 1983. Effects of "jet lag" on hormonal patterns. IV. Time shifts increase growth hormone release. J. Clin. Endocrinol. Metab. 56:433-440.

20. Refetoff, S., and P. H. Sonksen. 1970. Disappearance rate of endogenous and exogenous human growth hormone in man. J. Clin. Endocrinol. Metab. 30:386-392.

21. Turek, F. W. 1985. Circadian neural rhythms in mammals. Annu. Rev. Physiol. 47:49-64.

22. Ho, K. Y., J. D. Veldhuis, M. L. Johnson, R. Furlanetto, W. S. Evans, K. G. M. M. Alberti, and M. O. Thorner. 1988. Fasting enhances growth hor- mone secretion and amplifies the complex rhythms of growth hormone secretion in man. J. Clin. Invest. 81:968-975.

23. Vance, M., and M. Thorner. 1989. Fasting alters pulsatile and rhythmic cortisol release in normal man. J. Clin. Endocrinol. Metab. 68:1013-1018.

24. Linsell, C. R., S. L. Lightman, P. E. Mullen, M. J. Brown, and R. C. Causon. 1985. Circadian rhythms of epinephrine and norepinephrine in man. $J$. Clin. Endocrinol. Metab. 60:1210-1215.

25. Weitzman, E. D., J. C. Zimmerman, C. A. Czeisler, and J. Ronda. 1983. Cortisol secretion is inhibited during sleep in normal man. J. Clin. Endocrinol. Metab. 56:352-358.

26. Reaven, G., C. Hollenbeck, C. Jeng, M. S. Wu, and Y. I. Chen. 1988. Measurement of plasma glucose free fatty acid, lactate, and insulin for $24 \mathrm{~h}$ in patients with NIDDM. Diabetes. 37:1020-1024.

27. Polonsky, K. S., B. D. Given, and E. Van Cauter. 1988. Twenty-four-hou profiles and pulsatile patterns of insulin secretion in normal and obese subjects. $J$. Clin. Invest. 81:442-448.

28. Clore, J., J. Nestler, and W. Blackard. 1989. Sleep-associated fall in glucose disposal and hepatic glucose output in normal humans. Diabetes. 38:285290.

29. Ørskor, L., O. Schmitz, J. O. L. Jørgensen, J. Arnfred, N. Abildgaard, J. S Christiansen, K. G. M. M. Alberti, and H. Ørskov. 1989. Influence of growth hormone on glucose-induced glucose uptake in normal men as assessed by the hyperglycemic clamp technique. J. Clin. Endocrinol. Metab. 68:276-282.

30. Møller, N., P. C. Butler, M. A. Antsiferov, and K. G. M. M. Alberti. 1989. Effects of growth hormone on insulin sensitivity and forearm metabolism in normal man. Diabetologia. 32:105-110.

31. Vaitkus, P., A. Sirek, K. H. Norwich, O. V. Sirek, R. H. Unger, and V. Harris. 1984. Rapid changes in hepatic glucose output after a pulse of growth hormone in dogs. Am. J. Physiol. 246:E14-E20. 